\title{
PRIME IDEAL STRUCTURE OF RINGS OF BOUNDED CONTINUOUS FUNCTIONS
}

\author{
MARK MANDELKER
}

Introduction. The order structure of the family of prime ideals in the ring $C$ of all real-valued continuous functions on a topological space has been extensively studied; in this paper we study the analogous problem in the subring $C^{*}$ of bounded functions. The fundamental property of prime ideals in $C^{*}$ is the following.

Main Theorem. Let $M^{*}$ be any maximal ideal of $C^{*}$ and let $M$ be the unique maximal ideal of $C$ such that the prime ideal $M \cap C^{*}$ is contained in $M^{*}$. Then every prime ideal contained in $M^{*}$ is comparable with $M \cap C^{*}$.

The proof involves topological properties of the Stone-Cech compactification $\beta X$ of a completely regular Hausdorff space $X$.

Of special interest are the prime $z$-ideals of $C^{*}$. When $X$ is a locally compact, $\sigma$-compact Hausdorff space, we show that the family of prime $z$-ideals of $C^{*}(X)$ contained in $M^{*}$ is composed of two subfamilies, order-isomorphic with naturally corresponding families of prime $z$-ideals in the rings $C(X)$ and $C(\beta X-X)$.

1. Preliminaries. We shall use the terminology and notation of the Gillman-Jerison text [3]. Applying [3, Theorem 3.9], we immediately reduce the problem of the prime ideal structure of $C^{*}(X)$, and its relation to $C(X)$, to the case that $X$ is a completely regular Hausdorff space. A basic property of prime ideals in rings of functions that will be used several times is a theorem of Kohls ([9, Theorem 2.4], see also $[3,14.8(\mathrm{a}), 6.6(\mathrm{c})])$ : In the ring $C(X)$, and also in $C^{*}(X)$, the prime ideals containing a given prime ideal form a chain.

The proof of the main theorem is based on Kohls' result and the following theorem $([10,4.4]$; cf. $[7,3.1]$ and [6, p. 112]): A prime $z$-filter $\mathcal{Q}$ on a space $T$ is minimal if and only if for every zero-set $Z$ in $Q$ there is a zero-set $W$ not in $Q$ such that $Z \cup W=T$.

We shall use $\mathfrak{M}^{p}$ and $\boldsymbol{O}^{p}$ to denote the $z$-filters $Z\left[M^{p}\right]$ and $Z\left[O^{p}\right]$, respectively.

2. The main theorem. Under the reduction made in $\$ 1$ to the case of a completely regular Hausdorff space $X$, a maximal ideal of $C^{*}(X)$ corresponds to a point $p$ of $\beta X$ and is denoted $M^{* p}$, and the maximal ideal $M^{p}$ of $C(X)$ that corresponds to $p$ is the unique maximal ideal

Received by the editors July 28, 1967. 
of $C(X)$ such that $M^{p} \cap C^{*} \subseteq M^{* p}$ (see [3, Chapter 7]). Thus the main theorem takes the following form.

Theorem I. Let $p \in \beta X$. Every prime ideal $P$ of $C^{*}(X)$ contained in $M^{* p}$ is comparable with $M^{p} \cap C^{*}$. Specifically, $P \subseteq M^{p} \cap C^{*}$ if and only if $P$ contains no unit of $C$, while $M^{p} \cap C^{*} \subset P$ if and only if $P$ contains a unit of $C$.

Proof. Let $P$ be any prime ideal of $C^{*}(X)$ with $P \subseteq M^{* p}$. Choose a minimal prime ideal $Q$ with $Q \subseteq P$. To prove that $P$ and $M^{p} \cap C^{*}$ are comparable, it suffices to show that $Q \subseteq M^{p} \cap C^{*}$, for then $P$ and $M^{p} \cap C^{*}$ both contain the prime ideal $Q$.

To show that $Q \subseteq M^{p} \cap C^{*}$, we first pass to the $\operatorname{ring} C(\beta X)$ by means of the canonical isomorphism $f \rightarrow f^{\beta}$ of $C^{*}(X)$ onto $C(\beta X)$ $[3,6.6(\mathrm{~b})]$, and then we pass to the family of prime $z$-filters on $\beta X[3,2.12]$. According to the Gelfand-Kolmogoroff theorem $[3,7.3]$, the prime ideal in $C(\beta X)$ corresponding to $M^{p} \cap C^{*}$ is given by

$$
\left(M^{p} \cap C^{*}\right)^{\beta}=\left\{g \in C(\beta X): p \in \operatorname{cl}_{\beta X} Z_{X}(g \mid X)\right\} .
$$

Since $\boldsymbol{Z}_{X}(g \mid X)=\boldsymbol{Z}_{\beta X}(g) \cap X$, this is a $z$-ideal in $C(\beta X)$. We denote the corresponding prime $z$-filter on $\beta X$ by $\mathscr{N}^{p}$; thus

$$
\mathfrak{T}^{p}=\left\{Z \in Z(\beta X): p \in \operatorname{cl}_{\beta X}(Z \cap X)\right\} .
$$

Also, the minimal prime ideal $Q^{\beta}$ of $C(\beta X)$ corresponding to $Q$ is a $z$-ideal $[3,14.7]$; we denote the corresponding minimal prime $z$-filter on $\beta X$ by $Q$. Let $Z \in Q$ and let $V$ be any zero-set-neighborhood of $p$ in $\beta X$. Since $Q \subseteq \mathfrak{N}_{\beta X}^{p}$ and $V \in \mathcal{O}_{\beta X}^{p}$ we have $V \in \mathcal{Q}[3,7.15]$ and thus $V \cap Z \in Q$. Using the minimality of $Q$, we choose a zero-set $W$ not in $Q$ such that $(V \cap Z) \cup W=\beta X$. If $V \cap Z$ has empty interior in $\beta X$, then $W$ is dense in $\beta X$; so $W=\beta X$ and $W \in Q$, contradicting the choice of $W$. Hence $V \cap Z$ has nonempty interior in $\beta X$, and thus $(V \cap Z) \cap X \neq \varnothing$. This shows that every neighborhood of $p$ in $\beta X$ meets $Z \cap X$; hence $p \in \operatorname{cl}_{\beta X}(Z \cap X)$ and $Z \in \mathscr{T}^{p}$. Thus $Q \subseteq \mathscr{T}^{p}$ and it follows that $Q \subseteq M^{p} \cap C^{*}$.

Now assume that $P$ contains no unit of $C$. Let $f \in P$ and let $V$ be any zero-set-neighborhood of $p$ in $\beta X$. Since $Z\left[P^{\beta}\right]$ is a prime $z$-filter on $\beta X$ contained in $\mathfrak{T}_{\beta X}^{p}$, we have $V \in \boldsymbol{Z}\left[P^{\beta}\right]$, so that $V \cap \boldsymbol{Z}\left(f^{\beta}\right)$ $\in \boldsymbol{Z}\left[P^{\beta}\right]$ and thus also $V \cap \boldsymbol{Z}(f) \in \boldsymbol{Z}[P]$. Since $P$ contains no unit of $C$, $V \cap \boldsymbol{Z}(f) \neq \varnothing$. Hence $p \in \operatorname{cl}_{\beta X} \boldsymbol{Z}(f)$, i.e., $f \in \boldsymbol{M}^{p}$. Thus $P \subseteq \boldsymbol{M}^{p} \cap C^{*}$. The converse is immediate, and the last statement then follows from the comparability.

REMARKs. The second part of the theorem generalizes $[3,7.9]$ : $M^{* p}=M^{p} \cap C^{*}$ if and only if $M^{* p}$ contains no unit of $C$. 
We also note that a nonunit of $C$ in $M^{* p}$ need not be contained in $M^{p} \cap C^{*}$. For example, choose any function $g$ in $C^{*}(\mathrm{R})$ that vanishes at infinity and has nonempty compact zero-set. Then $g$ is a nonunit of $C$ and for any $p \in \beta \mathrm{R}-\mathrm{R}$, we have $g \in M^{* p}$ but $g \notin M^{p} \cap C^{*}$. Whenever $f^{\beta}(p)=0, \boldsymbol{Z}(f) \neq \varnothing$, but $p \in \mathrm{cl}_{\beta X} \boldsymbol{Z}(f)$, Theorem I shows that although $f \in M^{* p}$ and $f$ is a nonunit of $C, M^{* p}$ contains no prime ideal that contains $f$ and contains only nonunits of $C$.

3. $z$-ideals in $C^{*}$. As in $C$, a $z$-ideal in $C^{*}$ is an ideal $I$ that contains any function that belongs to the same maximal ideals as some function in $I$ (see $[8$, p. 30] and $[3,2.7,4 \mathrm{~A} .5])$. Thus the $z$-ideals of $C^{*}(X)$ are the ideals that correspond to z-ideals of $C(\beta X)$ under the isomorphism $f \rightarrow f^{\beta}$, and the family of all prime $z$-ideals of $C^{*}(X)$ is orderisomorphic with the family of all prime $z$-filters on $\beta X[3,2.12]$.

Every minimal prime ideal in $C$ is a $z$-ideal $[3,14.7]$. Thus a prime ideal in $C$ is minimal if it contains no prime $z$-ideals. Also, if the prime $z$-ideals contained in a given maximal ideal of $C$ form a chain, then all the prime ideals contained in that maximal ideal form a chain. By $[3,6.6(\mathrm{c})]$, prime ideals in $C^{*}$ also have these properties.

4. The isomorphism theorem. In the case that $\beta X-X$ is a zero-set in $\beta X$ (equivalently, that $X$ is locally compact and $\sigma$-compact), the prime $z$-ideal structure of $C^{*}(X)$ may be described entirely in terms of prime $z$-ideals in the rings $C(X)$ and $C(\beta X-X)$. Some of the known results on the structure of these rings will be applied in $\$ \S 6$ and 7 to obtain information on the structure of $C^{*}(X)$.

When $X$ is locally compact and $\sigma$-compact, there is a bounded unit of $C$ that belongs to $M^{* p}$ for every $p \in \beta X-X$; thus $M^{p} \cap C^{*} \neq M^{* p}$ if and only if $p \notin X$.

THEOREM II. Let $X$ be locally compact and $\sigma$-compact, and let $p \in \beta X$.

(a) The family of prime z-ideals of $C^{*}(X)$ contained in $M^{p} \cap C^{*}$ is order-isomorphic with the family of prime z-ideals of $C(X)$ contained in $M^{p}$.

(b) The family of prime z-ideals of $C^{*}(X)$ properly containing $M^{p} \cap C^{*}$ (when $\left.p \notin X\right)$ is order-isomorphic with the family of prime $z$-ideals of $C(\beta X-X)$ contained in $M_{\beta X-X}^{p}$.

Proof. We first place the prime $z$-ideals contained in $M^{* p}$ in orderpreserving correspondence with the prime $z$-filters on $\beta X$ contained in $\Re_{\beta X}^{p}$, by means of the mapping $P \rightarrow \boldsymbol{Z}\left[P^{\beta}\right]$. Under this mapping, we have $M^{p} \cap C^{*} \rightarrow \mathfrak{T}^{p}$ (see $\S 2$ ). The order-isomorphisms will now be obtained by means of traces and induced $z$-filters $[10, \S 5]$.

(a) If $\mathcal{P}$ is a prime $z$-filter contained in $\mathfrak{x}^{p}$, then every member of 
P meets $X$. By [10, Theorem 5.2], the trace

$$
\odot \mid X=\{Z \cap X: Z \in \odot\}
$$

of $\mathcal{P}$ on $X$ is a prime $z$-filter on $X$. Since $\mathcal{P} \subseteq \mathfrak{I}^{p}$, we have $\mathcal{P} \mid X \subseteq \mathfrak{M}_{X}^{p}$. If $\mathcal{Q}$ is any prime $z$-filter on $X$ contained in $\mathfrak{M}_{X}^{p}$, the induced prime $z$-filter

$$
Q^{\#}=\{Z \in Z(\beta X): Z \cap X \in Q\}
$$

is clearly contained in $\mathfrak{T}^{p}$, and $\Theta^{\sharp} \mid X=\Theta$. Hence the mapping $\odot \rightarrow \odot \mid X$, for $P \subseteq \mathscr{N}^{p}$, is onto the family of prime $z$-filters on $X$ contained in $\mathfrak{M}_{X}^{p}$. For any $\mathcal{P} \subseteq \mathfrak{N}^{p}$, it is immediate that $\mathcal{P} \subseteq(\mathcal{P} \mid X)^{*}$. Now let $Z \in(\odot \mid X)^{\sharp}$; thus there is $W \in \odot$ such that $Z \cap X=W \cap X$. Since

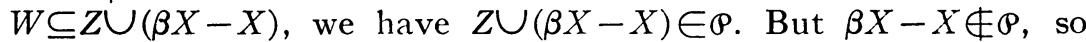

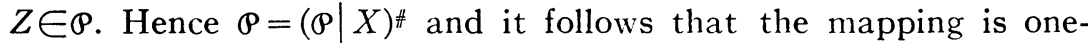
to-one.

(b) If $\beta$ is a prime $z$-filter on $\beta X$ properly containing $\mathscr{T}^{p}$, then by $[10$, Theorem 5.2], the trace $\beta \mid(\beta X-X)$ is a prime $z$-filter on $\beta X-X$. Since $\mathcal{P} \subseteq \mathfrak{M}_{\beta X}^{p}$, we have $\beta \mid(\beta X-X) \subseteq \mathfrak{M}_{\beta X-X}^{p}$. If $Q$ is any prime $z$-filter on $\beta X-X$ contained in $\mathfrak{T}_{\beta X-X}^{p}$, the induced $z$-filter

$$
\mathrm{Q}^{\#}=\{Z \in Z(\beta X): Z \cap(\beta X-X) \in \mathcal{Q}\}
$$

is prime and $\mathcal{Q}^{f} \mid(\beta X-X)=\mathcal{Q}$. Since $\mathcal{Q} \subseteq \mathfrak{M}_{\beta X-X}^{p}$, we have $\mathcal{Q}^{\sharp} \subseteq \mathfrak{M}_{\beta X}^{p}$. Furthermore, the zero-set $\beta X-X$ is in $Q^{\#}$ but clearly not in $\mathfrak{x}^{p}$; hence $\mathcal{Q}^{\#} \Phi \mathfrak{N}^{p}$. It now follows from Theorem I that $\mathcal{Q}^{\#}$ properly contains $\mathscr{T}^{p}$. Thus the mapping $\mathcal{P} \rightarrow \mathcal{P} \mid(\beta X-X)$, for $\mathscr{T}^{p} \subset \mathcal{P}$, is onto the family of prime $z$-filters on $\beta X-X$ contained in $\mathfrak{T}_{\beta X-X}^{p}$. It is clear that $\rho \subseteq(P \mid(\beta X-X))^{\sharp}$. Now let $Z \in(\rho \mid(\beta X-X))^{\sharp}$; thus there is $W \in \beta$ such that $Z \cap(\beta X-X)=W \cap(\beta X-X)$. By Theorem I the

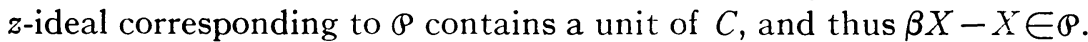
It follows that $Z \in \odot$. Hence $\odot=(\odot \mid(\beta X-X))^{\#}$ and the mapping is one-to-one.

5. An application to $F$-spaces. An immediate consequence of Theorem II is a well-known theorem on $F$-spaces $[2,2.7]$, (see also [3, 14.27] and [11,3.3]). A space $T$ is an $F$-space if every finitely generated ideal in $C(T)$ is principal, or, equivalently, if the prime ideals contained in any given maximal ideal form a chain $[3,14.25]$. In part (b) of Theorem II, the prime z-ideals properly containing the prime ideal $M^{p} \cap C^{*}$ form a chain; thus we have

Corollary 1 (Gillman-Henriksen). If $X$ is locally compuct and $\sigma$-compact, then $\beta X-X$ is a compact F-space. 
6. Immediate successors. It was shown in $[4$, p. 432] that if $X$ is locally compact and $\sigma$-compact, and $p \in \beta X-X$, then $\mathscr{T}^{p}$ has an immediate successor $\left(\Re^{p}\right)^{+}$in the family of prime $z$-filters on $\beta X$, generated by $\mathfrak{T}^{p}$ and the zero-set $\beta X-X$, i.e.

$$
\left(\mathscr{T}^{p}\right)^{+}=\left(\mathscr{T}^{p}, \beta X-X\right) .
$$

(This result may also be obtained from Theorem I, which shows that a prime $z$-filter contained in $\mathfrak{T}_{\beta X}^{p}$ properly contains $\mathfrak{T}^{p}$ if and only if it contains the zero-set $\beta X-X$.)

Furthermore, it is shown in $[4$, p. 433$]$ that in the case of the countably infinite discrete space $\mathbf{N}$,

$$
\left(\Re^{p}\right)^{+}=\left(\mathcal{O}_{\beta \mathbf{N}}^{p}, \beta \mathbf{N}-\mathbf{N}\right) .
$$

We now generalize this as follows.

Corollary 2. Let $X$ be locally compact and $\sigma$-compact, and let $p \in \beta . X-X$. Then

$$
\left(\mathscr{\Re}^{p}\right)^{+}=\left(\Theta_{\beta X}^{p}, \beta X-X\right) .
$$

Hence the immediate successor of $\boldsymbol{M}^{p} \cap C^{*}$ in the family of prime z-ideals of $C^{*}(X)$ consists of all functions $f$ such that $f^{\beta}$ vanishes on a neighborhood of $p$ in $\beta X-X$.

Proof. According to the construction of the second isomorphism in $\S 4$, we have $\left(\mathscr{T}^{p}\right)^{+}=\left(\mathcal{O}_{\beta X-X}^{p}\right)^{\#}$, and it is easily verified that

$$
\left(\mathcal{O}_{\beta X}^{p}, \beta X-X\right)=\left(\mathcal{O}_{\beta X-X}^{p}\right)^{f} .
$$

REMARK. The present paper began with the observation that although $\Theta_{\beta \mathrm{R}}^{p}$ is usually not prime (see [10, Theorem 11.2]), the $z$-filter $\left(\Theta_{\beta R}^{p}, \beta \mathbf{R}-\mathbf{R}\right)$ is always prime, because of the above representation as an induced $z$-filter and the Gillman-Henriksen theorem of $\$ 5$. This raised the question of its relation to $\mathscr{T}^{p}$ and $\left(\mathscr{T}^{p}, \beta R-R\right)$.

7. Remote points and $P$-points. For any space $X$, a point $p$ in $\beta X$ is called a remote point in $\beta X$ if every member of $\mathfrak{M}_{X}^{p}$ has nonempty interior (see [1]). When $X$ is a metric space, remote generalizes isolated: a point $p$ in $X$ itself is a remote point in $\beta X$ if and only if it is an isolated point of $X$. Also, if $D$ is a discrete space, every point in $\beta D$ is a remote point in $\beta D$. When $X$ is a metric space with no isolated points, a point $p$ in $\beta X$ is a remote point in $\beta X$ if and only if $p$ is in the closure of no discrete subset of $X$ (see $[5, \S 23$, VIII $]$ ). 
Under the continuum hypothesis, the existence of remote points in $\beta \mathrm{R}$ was shown in [1]. It is shown in Theorem 11.2 of [10] (the proof given there for the real line is also valid for the case considered here) that if $X$ is a separable metric space and $p \in \beta X$, the following are equivalent: (a) The prime ideals contained in $M_{X}^{p}$ form a chain. (b) $M_{X}^{p}$ is a minimal prime ideal. (c) $p$ is a remote point in $\beta X$.

A point $p$ of a space $T$ is a $P$-point of $T$ if every zero-set containing $p$ is a neighborhood of $p[3,4 \mathrm{~L}]$; equivalently, if $\boldsymbol{M}_{T}^{p}$ is a minimal prime ideal $[3,14.12]$. Under the continuum hypothesis, there exist $P$-points of $\beta X-X$ whenever $X$ is locally compact but not pseudocompact $[3,9 \mathrm{M}]$.

Assuming the continuum hypothesis, Donald Plank [12, Theorem 6.2] has recently discovered points in $\beta R-R$ that are both remote points in $\beta \mathrm{R}$ and $P$-points of $\beta \mathrm{R}-\mathrm{R}$, points that are remote points but not $P$-points, points that are $P$-points but not remote points, and also points that are neither. He has also shown that each of these four classes of points is a dense subset of $\beta R-R$ of cardinal $2^{c}$. These points provide examples for the various types of prime ideal structure of $C^{*}$ described below.

Applying Theorems I and II, and Theorem 11.2 of [10] (as stated above), we obtain the following relations between points in $\beta X$ and the prime ideal structure of $C^{*}(X)$. (Corollary 4 generalizes [4, Theorem 3.10], which gives the result for the case $X=\mathbf{N}$.)

Corollary 3. Let $X$ be a locally compact, $\sigma$-compact metric space, and let $p \in \beta X$. Then the following conditions are equivalent.

(a) The prime ideals of $C^{*}$ contained in $M^{* p}$ form a chain.

(b) $M^{p} \cap C^{*}$ is a minimal prime ideal of $C^{*}$.

(c) $p$ is a remote point in $\beta X$.

Corollary 4. Let $X$ be locally compact and $\sigma$-compact, and let $p \in \beta X-X$. Then $M^{* p}$ is the immediate successor of $M^{p} \cap C^{*}$ in the family of prime $z$-ideals of $C^{*}(X)$ if and only if $p$ is a $P$-point of $\beta X-X$.

Corollary 5. Let $X$ be a locally compact, $\sigma$-compact metric space, and let $p \in \beta X-X$. Then the family of prime z-ideals of $C^{*}$ contained in $M^{* p}$ consists of just the two ideals $M^{* p}$ and $\boldsymbol{M}^{p} \cap C^{*}$ if and only if $p$ is both a remote point in $\beta X$ and a P-point of $\beta X-X$.

\section{REFERENCES}

1. N. J. Fine and L. Gillman, Remote points in $\beta$ R, Proc. Amer. Math. Soc. 13 (1962), 29-36.

2. L. Gillman and M. Henriksen, Rings of continuous functions in which every finitely generated ideal is principal, Trans. Amer. Math. Soc. 82 (1956), 366-391. 
3. L. Gillman and M. Jerison, Rings of continuous functions, Van Nostrand, Princeton, N. J., 1960.

4. - Quotient fields of residue class rings of function rings, Illinois J. Math. $4(1960), 425-436$.

5. F. Hausdorff, Mengenlehre, 3rd ed., Göschens Lehrbuch. 7, de Gruyter, Berlin, 1935.

6. M. Henriksen and M. Jerison, The space of minimal prime ideals of a commutative ring, Trans. Amer. Math. Soc. 115 (1965), 110-130.

7. J. Kist, Minimal prime ideals in commutative semigroups, Proc. London Math. Soc. (3) 13 (1963), 31-50.

8. C. W. Kohls, Ideals in rings of continuous functions, Fund. Math. 45 (1958), $28-50$.

9. - - Prime ideals in rings of continuous functions, Illinois $\mathrm{J}$. Math. 2 (1958), $505-536$.

10. M. Mandelker, Prime z-ideal structure of $C(\mathbf{R})$, Fund. Math. 63 (1968), 145-166.

11. S. Negrepontis, Absolute Baire sets, Proc. Amer. Math. Soc. 18 (1967), 691694.

12. D. Plank, On a class of subalgeoras of $C(X)$ with applications to $\beta X-X$, Fund. Math. (to appear).

University of Kansas 